\title{
A CHARACTERIZATION OF INVARIANT AFFINE CONNECTIONS
}

\author{
BERTRAM KOSTANT
}

1. Introduction and statement of theorem. 1. In [1] Ambrose and Singer gave a necessary and sufficient condition (Theorem 3 here) for a simply connected complete Riemannian manifold to admit a transitive group of motions. Here we shall give a simple proof of a more general theorem-Theorem 1 (the proof of Theorem 1 became suggestive to us after we noted that the $T_{x}$ of [1] is just the $a_{x}$ of [6] when $X$ is restricted to $p_{0}$, see [6], p. 539). In fact after introducing, below, the notion of one affine connection $A$ on a manifold being rigid with respect to another affine connection $B$ on $M$ and making some observations concerning such a relationship, Theorem 1 is seen to be a reformulation of Theorem 2. But Theorem 2 may be obtained as a consequence of some work of Nomizu and Kobayashi. ${ }^{1)}$ Here we refer especially to the work of these mathematicians on the theory of affine connections which are invariant under parallelism. Such an affine connection was called locally reductive in [4]. (Since a slight elaboration of this theory was needed for our purposes, rather than "fill in", we have preferred instead to give a somewhat different, almost self-contained, account of the relevant portion of this theory here.)

Without any statement to the contrary it will be assumed throughout this paper that the manifold $M$ and any affine connection or tensor field to be considered on $M$ is of class $C^{\infty}$.

1.2. We shall need some definitions.

(a) The notion of a reductive homogeneous space was introduced by Nomizu [7]. Assume that $G$ is a connected Lie group and $G$ is given as operating transitively on a manifold $M$ as a group of homeomorphisms in such a way that the map $G \times M \rightarrow M$ defined by $(g, o) \rightarrow g \cdot o$ is of class $C^{\infty}$. Here $g \cdot o$ is the image of $o \in M$ under the action of $g \in M$. Let $\mathfrak{g}$ be the Lie algebra of

Received August 13, 1959.

1) We have been informed by correspondence that Nomizu has also obtained a similar generalization of Theorem 3 . 
$G$. We then say that $M$ is a reductive homogeneous space with respect to the action of $G$ if for some point $o \in M$ (and consequently for every point of $M$ ) the subgroup $H$ of all elements of $G$ which leave $o$ fixed (the isotropy group at $o$ ) has the following property. If $\mathfrak{h} \subseteq \mathrm{g}$ is the Lie algebra of $H$ then there exists a subspace $\mathfrak{m} \subseteq \mathfrak{g}$ such that (1) $\mathfrak{g}=\mathfrak{h}+\mathfrak{m}$ is a direct sum and (2) $m$ is stable under AdH.

Note 1. The action of a connected Lie group $G$ (or in fact a local group) with Lie algebra $g$ (which we shall understand brackets like right invariant vector fields on $G$ ) on a manifold $M$ induces a homomorphism $\gamma$ of $g$ into the Lie algebra of all vector fields on $M$ by the relation $r\left(X_{1}\right)=X$ where $X_{1} \in g$ and

$$
(X f)_{p}=\left.\frac{d}{d t} f\left(\exp t X_{1} \cdot p\right)\right|_{t=0}
$$

where $p \in M$ and $f$ is any function on $M$. When there is no danger of confusion and $\gamma$ is faithful we will usually identify $g$ with $\gamma^{g}$ by the isomorphism $\gamma$.

(b) Let $B$ be an affine connection on a manifold $M$. Let $T^{B}$ and $R^{B}$ be, respectively, the torsion and curvature tensor fields on $M$ with respect to $B$. The affine connection $B$ will be called invariant under parallelism in case $T^{B}$ and $R^{B}$ are both covariant constant. (This is not the definition used by Nomizu. Nevertheless Theorem 18.1 in [7] implies that it is an equivalent definition.) Spaces with such an affine connection generalize locally symmetric spaces in that the torsion tensor is not assumed to vanish. However, as shown in [4] and [7] much of the formal aspects of symmetric space theory goes through. In particular, as in the symmetric case, one easily shows that such spaces have a "rich supply", locally, of infinitesimal affine transformations.

(c) Let $A$ and $B$ be any two affine connections on a manifold $M$. One knows that $A$ and $B$ differ by a tensor field $S$ of type $\left(\begin{array}{c}i \\ j k\end{array}\right)$. See $\S 2.2$ for more details. We will say that $A$ is rigid with respect to $B$ whenever $S$ is covariant constant with respect to $B$.

(d) Finally an affine connection $B$ on a manifold $M$ is said to be complete if every geodesic may be extended for arbitrary large values of its canonical parameter.

Theorem 1 which will be shown in $\$ 2$ to generalize the result of AmbroseSinger gives a geometric characterization of an affinely connected simply: 
connected manifold which admits a transitive group of affine transformations with respect to which it is a reductive homogeneous space.

THEOREM 1. Let $A$ be an affine connection on a simply connected manifold $M$. Then $M$ is a reductive homogeneous space with respect to a connected Lie group $G$ whose action leaves $A$ invariant if and only if there exists an affine connection $B$ on $M$ such that (1) $R^{A}$ and $T^{A}$ is covariant constant with respect to $B$, (2) $A$ is rigid with respect to $B$ and (3) $M$ is complete with respect to $B$.

Theorem 2 offers a characterization which says in effect that the affine connection should not differ very much from an affine connection which is invariant under parallelism.

THEOREM 2. Let $A$ be an affine connection on a simply connected manifold $M$. Then $M$ is a reductive homogeneous space with respect to a connected Lie group $G$ whose action leaves $A$ invariant if and only if there exists an affine connection $B$ on $M$ such that (1) $B$ is invariant under parallelism, (2) $A$ is rigid with respect to $B$ and (3) $M$ is complete with respect to $B$.

Theorem 2 will be proved in $\S 3$ and $\S 4$ as Theorems 4 and $\overline{5}$.

2. Preliminaries. 1. For $k=0,1, \ldots$ let $\mathscr{T}^{K}(M)$ be the space of all contravariant tensor fields of degree $k$ and class $C^{\infty}$ on $M$ and let $\mathscr{T}(M)$ be the algebra, $\mathscr{T}(M)=\sum_{k=0}^{\infty} \mathscr{T}^{k}(M)$. Let $\mathscr{D}(M)$ be the Lie algebra of all derivations of $\mathscr{T}(M)$ which preserve degree and which on the space of scalar functions $\mathscr{T}^{0}(M)$ reduces to differentiation by a vector field $X \in \mathscr{T}^{1}(M)$. In fact let

$$
\sigma: \mathscr{Z}(M) \rightarrow \mathscr{T}^{1}(M)
$$

be the mapping defined by setting $\sigma(D)=X \in \mathscr{T}^{1}(M)$ where $D \in \mathscr{L}(M)$ and $D f=X f$ for all $f \in \mathscr{T}^{0}(M)$. Let $\mathscr{L}(M)$ be the kernel of $\sigma$. Since an element in $\mathscr{L}(M)$ is determined by its action on $\mathscr{T}^{0}(M)$ and $\mathscr{T}^{1}(M)$ it is clear that we may identify $\mathscr{L}(M)$ with the space of all $C^{\text {» }}$ fields of tangent space endomorphisms.

Now if $A$ is an affine connection on $M, A$ sets up (and is given by) a $\mathscr{T}^{0}(M)$-linear map

$$
\pi_{A}: \mathscr{T}^{-1}(M) \rightarrow \mathscr{g}(M)
$$

where $\pi_{1}(X)=\Gamma_{X}^{1} \in \mathscr{S}^{\prime}(M)$ is covariant differentiation by $X$ with respect to $A$. 
Furthermore $\sigma \pi_{A}$ is the identity map on $\mathscr{T}^{1}(M)$.

2.2. Now if $A$ and $B$ are any two $C^{\nu}$ affine connections on $M$ then $A$ and $B$ differ by a tensor field $S$ of type $\left(\begin{array}{c}i \\ j k\end{array}\right)$ in the following sense. For any $X \in \mathscr{T}^{1}(M)$ let $S_{X} \in \mathscr{D}(M)$ be defined by

$$
S_{X}=\nabla_{\mathrm{X}}^{B}-\nabla_{X}^{A} .
$$

But clearly $S_{X} \in \mathscr{L}(M)$. The tensor field $S$ is then the $\mathscr{T}^{0}(M)$-linear map of $\mathscr{T}^{1}(M)$ into $\mathscr{L}(M)$ given by $X \rightarrow S_{X}$. When so defined we will write

$$
S=B-A \text {. }
$$

Conversely, if an affine connection $A$ and a tensor field $S$, defining a $\mathscr{T}^{0}(M)$-linear map of $\mathscr{T}^{1}(M)$ into $\mathscr{L}(M)$, are given then $A$ and $S$ define an affine connection $B$, where $B-A=S$, by the relation (2.2.1).

2.3. In [1] Ambrose and Singer proved the following theorem:

THEOREM 3. Let $M$ be a simply connected complete Riemannian manifold. Then the group (and hence its identity component) of motions of $M$ is transitive on $M$ if and only if there exists a tensor field $S\left(\right.$ of type $\left.\left(\begin{array}{c}i \\ j k\end{array}\right)\right)$ defining a $\mathscr{T}^{0}(M)$-linear map, $X \rightarrow S_{X}$, of $\mathscr{T}^{1}(M)$ into $\mathscr{L}(M)$ such that (1) $\left(S_{X}\right)_{p}$ is a skew-symmetric operator on the tangent space at $p \in M$ with respect to the metric tensor at $p$ for all $p \in M$ and $X \in \mathscr{T}^{1}(M)$ and satisfies; for $X, Y, Z \in \mathscr{T}^{1}(M)$,

(2) $\quad\left(\nabla_{X}^{A} R^{A}\right)(Y, Z)=R^{A}\left(S_{X} Y, Z\right)+R^{A}\left(Y, S_{X} Z\right)-\left[S_{X}, R^{A}(Y, Z)\right]$

and

(3) $\left.\left(\nabla_{X}^{A} S\right)_{Y}=S_{S_{X} Y}-\left[S_{X}, S_{Y}\right]\right)$.

(Here $A$ designates the Levi-Cevita connection with respect to the metric.)

We now observe that Theorem 3 is contained in Theorem 1 . We first note that (2) and (3) of Theorem 3 may be expressed, respectively, by the relations $\nabla_{X}^{A} R^{A}=-S_{X} R^{A}$ and $\nabla_{X}^{A} S=-S_{X} S$, or if the affine connection $B$ is defined by $B-A=S, \nabla_{X}^{B} R^{A}=0$ and $\nabla_{X}^{B} S=0$. That is, (a) $A$ is rigid with respect to $B$ and (b) $R^{A}$ is covariant constant with respect to $B$. Conversely if an affine connection $B$ exists which satisfies (a) and (b) clearly $S=B-A$ satisfies (2) and $(3)$.

Now assume that $S$ exists satisfying (1), (2), and (3) of Theorem 3. Let 
$B=A+S$. Since $T^{A}=0$ it is clear that $B$ satisfies (1) and (2) of Theorem 1 . We assert that $B$ also satisfies (3) of Theorem 1 . In fact by Lemma $3 B$ is invariant under parallelism and this follows from elementary facts concerning such connections. Indeed by Lemma 9 , and in the notation of Lemma 9 it suffices to show thet $g$ is the Lie algebra of $G$. That is, it suffices to show that any element of $g$ generates a one-parameter of transformations of $M$. But since $M$ is assumed complete with respect to the connection $A$ and since $B$ is a Lie algebra of infinitesimal $A$-affine transformations (see Lemma 8 ) one knows that every element of $g$ generates a one-parameter group. See Prop, p. 67 in [8]. We can now apply Theorem 1 which asserts among other things that there exists a connected Lie group which operates transitively on $M$ as a group of $A$-affine transformations. We wish to show the group of isometries is transitive on $M$. But by the de Rham decomposition $M$ is isometric to a direct product $M_{0} \times M_{1}$ where $M_{0}$ is isometric to Euclidean space and $M_{1}$ is simply connected, complete and is such that its holonomy group leaves no non-zero tangent vector fixed. It clearly suffices to show that the group of isometries of $M_{1}$ is transitive. But if $A_{1}$ is the Levi-Cevita connection on $M_{1}$ by Theorem 1, p. 104, in [3] the group of $A_{1}$-affine transformations on $M_{1}$ is clearly transitive. On the other hand, by Theorem 1 in [3] and the Theorem, p. 39, in [5] any $A_{1}$. affine transformation on $\mathrm{M}_{1}$ is an isometry.

Now conversely assume the group of motion is transitive on $M$. It is clear then that $M$ is a reductive homogeneous space with respect to a group whose action leaves $A$-invariant (See [6], p. 539 for a canonical complement $\left(p_{0}\right)$ to the Lie algebra of the isotropy group). Applying Theorem 1 there exists an affine connection $B$ on $M$ satisfying the three conditions of Theorem 1 . Let $S=B-A$. Then as we have noted above $S$ satisfies (2) and (3) of Theorem 3 . By now by Lemma $3 B$ is invariant under parallelism. Let $g$ be defined by (3.3.1). Now assume that the $A$-holonomy group leaves no non-zero tangent vector fixed. Applying Theorem 1 in [3] and the Theorem, p. 39 in [5] again the metric tensor on $M$ is invariant under $g$ and hence is covariant constant with respect to $B$ by Lemma 7. But then obviously $S$ also satisfies (1) of Theorem 1. For the general case consider, as above, the de Rham decomposition $M=M_{0} \times M_{1}$. The argument above applies to $M_{1}$ and asserts the existence of $S_{1}$ on $M_{1}$ which satisfies (1), (2) and (3) of Theorem 3. But if $S$ is the trivial 
extension of $S_{1}$ to $M$ it is obvious that $S$ also satisfies (1), (2) and (3) of Theorem 3.

Note that the above argument shows condition (1) in Theorem 3 can be eliminated.

2.4. Now given $S=B-A$ we let $S^{*}$ denote the tensor field (also of type $\left.\left(\begin{array}{c}i \\ j k\end{array}\right)\right)$ which defines the alternating $\mathscr{T}^{0}(M)$-bilinear map of $\mathscr{T}^{1}(M)$ into $\mathscr{T}^{1}(M)$ given by

$$
S^{*}(X, Y)=S_{X} Y-S_{Y} X .
$$

(In effect $S^{*}$ is obtained from $S$ by skew-symmetrizing the lower 2 indices of $S$.)

If $A$ is an affine connection on $M$ we will let $T^{A}$ and $R^{A}$ denote the corresponding torsion and curvature tensors. One knows that $T^{A}$ and $R^{A}$ define (and may in turn be defined by) alternating $\mathscr{T}^{0}(M)$-bilinear maps of $\mathscr{T}^{1}(M)$ into $\mathscr{T}^{1}(M)$ and $\mathscr{T}^{1}(M)$ into $\mathscr{L}(M)$ given by

$$
\begin{aligned}
& T^{A}(X, Y)=\nabla_{X}^{A} Y-\nabla_{Y}^{A} X-[X, Y] \\
& R^{A}(X, Y)=\left[\nabla_{X}^{A}, \nabla_{Y}^{A}\right]-\nabla_{[X, Y]}^{A} .
\end{aligned}
$$

We need some computational lemmas

Lemma 1. Let $A$ and $B$ be two affine connections on $M$. Let $S=B-A$ and let $S^{*}$ be defined by (2.4.1). Then

$$
T^{B}=T^{A}+S^{*} .
$$

Proof. Let $X, Y \in \mathscr{T}^{1}(M)$. By (2.4.1) and (2.4.2)

$$
\nabla_{X}^{B} Y-\nabla_{Y}^{H} X=\nabla_{X}^{A} Y-\nabla_{Y}^{A} X+S^{*}(X, Y) .
$$

Subtracting $[X, Y]$ from both sides it follows that

$$
T^{B}(X, Y)=T^{-1}(X, Y)+S^{*}(X, Y) .
$$

Corollary 1. Assume that $A$ is rigid with respect to $B$. Then $T^{A}$ is covariant constant with respect to $B$ if and only if $T^{B}$ is covariant constant with respect to $B$.

Proof. Follows immediately from Lemma 1.

Q.E.D.

Lemma 2. Let $A, B$, and $S$ be as in Lemma 1. Assume now that $A$ is rigid with respect to $B$. Then for any $X, Y \in \mathscr{T}^{1}(M)$ 


$$
R^{l}(X, Y)=R^{A}\left(X, Y^{\prime}\right)-\left(\left[S_{X}, S_{\zeta}\right]-S_{T^{l}(X, Y)}\right) .
$$

Proof. By (2.2.1)

$$
\begin{aligned}
{\left[\nabla_{X}^{A}, \nabla_{Y}^{A}\right] } & =\left[\nabla_{X}^{R}-S_{X}, \nabla_{Y}^{B}-S_{Y}\right] \\
& =\left[\nabla_{X}^{R}, \nabla_{Y}^{B}\right]+\left[S_{X}, S_{Y}\right]-\left(\nabla_{X}^{B}\left(S_{Y}\right)-\nabla_{Y}^{B}\left(S_{X}\right)\right)
\end{aligned}
$$

since $\left[\nabla_{X}^{B}, S_{Y}\right]=\nabla_{X}^{P}\left(S_{Y}\right)$ and similarly when $X$ and $Y$ are interchanged. But since $S$ is covariant constant with respect to $B$

$$
\Delta_{X}^{P}\left(S_{Y}\right)-\nabla_{Y}^{B}\left(S_{X}\right)=S_{\nabla_{X}^{B}}^{B} Y-\nabla_{Y}^{B} X
$$

On the other hand

$$
\nabla_{[X, Y]}^{A}=\nabla_{[X, Y]}^{B}-S_{[X, Y]} .
$$

Subtracting (2.4.5) from (2.4.4) it follows from (2.4.2) and (2.4.3) that

$$
R^{A}(X, Y)=R^{B}(X, Y)+\left[S_{X}, S_{Y}\right]-S_{T^{B}(X, 1)}
$$

Q.E.D.

Now Corollary 1 and Lemma 2 imply

Lemma 3. Assume that $A$ is rigid with respect to $B$. Then $T^{A}$ and $R^{4}$ are covariant constant with respect to $B$ if and only if $B$ is invariant under parallelism.

Lemma 3 proves that the statements of Theorems 1 and 2 are equivalent. Theorem 2 will be proved in the following sections.

3. Affine connections which are invariant under parallelism. 1. Let $M$ be a manifold and let $X \in \mathscr{T}^{1}(M)$. Let $L_{X} \in \mathscr{I}(M)$ designate Lie differentiation with respect to $X$. One has $L_{X} f=X f$ for any $f \in \mathscr{T}^{0}(M)$ so that $\sigma\left(L_{X}\right)=X$ and for any $Y \in \mathscr{T}^{1}(M)$

$$
L_{X} Y=[X, Y]
$$

Now assume $B$ is an affine connection on $M$. Let $X \in \mathscr{G}^{1}(M)$ and let $B_{X} \in \mathscr{Y}(M)$ be defined by

$$
B_{X}=L_{X}-\nabla_{X}^{l}
$$

Since $\sigma\left(L_{X}\right)=\sigma\left(\nabla_{X}^{B}\right)=X$ it follows that $B_{X}$ is a field of tangent space endomorphisms, that is $B_{X} \in \mathscr{S}(M)$.

Now let $Y \in \mathscr{T}^{1}(M)$. A simple straight forward computation using (2.4.2), (3.1.1) and (3.1.2) yields the relation 


$$
\nabla_{Y}^{B} X=T^{B}(X, Y)-B_{X} Y
$$

Now $X$ is an infinitesimal $B$-affine transformation if and only if for all $Y \in \mathscr{T}^{1}(M)$

$$
\left[L_{X}, \nabla_{Y}^{B}\right]=\nabla_{[X, 1]}^{R} .
$$

Again, one knows that $X$ is an infinitesimal $B$-affine transformation if and only if

$$
\nabla_{Y}^{B}\left(B_{X}\right)=R^{B}(X, Y)
$$

for all $Y \in \mathscr{T}^{1}(M)$. Indeed if (3.1.4) is satisfied then

$$
\begin{aligned}
\nabla_{Y}^{B}\left(B_{X}\right) & =\left[\nabla_{Y}^{B}, B_{X}\right] \\
& =\left[\nabla_{Y}^{B}, L_{X}-\nabla_{X}^{B}\right] \\
& =\left[\nabla_{X}^{B}, \nabla_{Y}^{B}\right]-\nabla_{X X, Y]}^{B} \\
& =R^{B}(X, Y)
\end{aligned}
$$

and conversely.

It follows immediately from the linear differential equations (3.1.3) and (3.1.5) that when $X$ is an infinitesimal $B$-affine transformation, the pair $X$ and $B_{X}$ are uniquely determined over $M$ by their values at one point. Let $g^{B}$ be the Lie algebra of all infinitesimal $B$-affine transformations. Let $X, Y \in \mathrm{g}^{I 3}$. It follows immediately from (3.1.2) and (3.1.3) that

$$
B_{X} Y-B_{Y} X+T^{B}(X, Y)=[X, Y] \text {. }
$$

On the other hand $\left[L_{X}, L_{Y}\right]=L_{[X, Y]}$ That is

$$
\left[\nabla_{X}^{B}+B_{X}, \nabla_{Y}^{R}+B_{Y}\right]=\nabla_{[X, Y]}^{B}+B_{[X, Y]} .
$$

Recalling that $\left[\nabla_{X}^{H}, B_{Y}\right]=\nabla_{X}^{R}\left(B_{Y}\right)$ (and again when $X$ and $Y$ are interchanged) it follows then from (3.1.5) and (3.1.7) that

$$
\left[B_{X}, B_{Y}\right]-R^{B}(X, Y)=B_{[X, Y]} .
$$

3.2. Now assume that $B$ is invariant under parallelism. It follows inmediately then from (2.4.3) that $B_{X} R^{B}=B_{X} T^{B}=0$ for all $X \in \mathrm{g}^{B}$. For any $p \in M$ let $a_{p}$ be the Lie algebra of all linear endomorphisms $b$ of the tangent space $V_{p}$ at $p$ such that $b\left(\left(R^{B}\right)_{p}\right)=b\left(\left(T^{B}\right)_{p}\right)=0$. It follows immediately from (3.1.8) that $R^{R}(u, v) \in \mathfrak{a}_{p}$ for all $u, v \in V_{p}$. It is clear that $R^{B}(u, v)$ and $T^{\prime}(u, v)$ for $u, v \in V_{p}$ are well defined since $R^{B}(X, Y)_{p}$ and $T^{B}(X, Y)_{p}$ depend only on $X_{p}, Y_{p}$. In fact it is clear that the set 


$$
\Xi_{p}=\left\{b \in a_{p} \mid b=\sum_{i} R^{B}\left(u_{i}, v_{i}\right), u_{i}, v_{i} \in V_{p}\right\}
$$

is an ideal of $a_{p}$. Now let $g_{p}^{B}$ be the direct sum of $a_{p}$ and $V_{p}$, to be written as the set of all pairs $(b, v)$ where $b \in a_{p}, v \in V_{p}$. The space $g_{p}^{B}$ is made into a Lie algebra by having the usual bracket in $a_{p}$, letting $[b, v]=b(v)=-[v, b]$ where $b \in a_{p}, v \in V_{p}$ and letting $[u, v]=\left(-R^{B}(u, v), T^{B}(u, v)\right)$. See [7], p. 62 for the proof of the Jacobi identity. The Lie algebra $g_{p}^{*}$ is obviously isomorphic to the Lie algebra defined by Nomizu on p. 62 in [7] under the isomorphism $(b, v) \rightarrow(b,-v)$. It follows then from (3.1.6) and (3.1.8) that if

$$
\alpha_{p}: g^{B} \rightarrow g_{p}^{B}
$$

is the linear mapping defined by $\alpha(X)=\left(\left(B_{X}\right)_{p}, X_{p}\right)$ then $\alpha_{p}$ is an isomorphism of $g^{B}$ into $g_{p}^{P}$.

3.3. Let $W$ be any open set in $M$ and let $\mathfrak{g}_{W}^{B}$ be the Lie algebra of all infinitesimal $B$-affine transformations defined on $W$. For $p \in W$ we may regard $\alpha_{p}$ as defined on $\oint_{W}^{B}$ by replacing $M$ by $W$ in the previous definition. In [7], p. 62 Nomizu has shown that if $W$ is a sufficiently small neighborhood of $p \in M$ then $\alpha_{p}$ defines an isomorphism of $g_{W}^{P}$ onto $g^{B}$ and furthermore $M$ is a locally $B$-affine homogeneous space with $g_{W}^{B}$ as the Lie algebra of the local group of $B$-affine transformations mapping $p$ into $W$. It follows then that $M$ has an underlying analytic structure and that the affine connection is analytic with respect to it. But then a theorem of Nijenhuis asserts that the Lie algebra of the restricted holonomy group at $p$ (the holonomy algebra at $p$ ) is obtained by contracting successive covariant derivatives of $R^{B}$ at $p$. Since $R^{B}$ is constant this implies (See [7], p. 50).

LeMma 4. Let $B$ be an affine connection which is invariant under parallelism on a manifold $M$. Let $p \in M$ and let the Lie algebra $\Xi_{p}$ of endomorphisms of the tangent space $V_{p}$ at $p$, be given by (3.2.1). Then $\mathfrak{s}_{p}$ is the holonomy algebra at $p$.

Let $X \in g^{B}$ and let $p \in M$. Then if $\left(B_{X}\right)_{p} \in \tilde{s}_{p}$ it follows from the equation (3.1.5) that $\left(B_{X}\right)_{q} \in \mathfrak{a}_{q}$ for any point $q \in M$. Let

$$
g=\left\{X \in \mathfrak{g}^{I} \mid\left(B_{X}\right\rangle_{0} \in g_{0} \text { at some (and hence any) point } o \in M\right\}
$$

and for any $p \in M$ and let $g_{p} \in g_{p}^{B 3}$ be the subspace spanned by $g_{p}$ and $V_{p}$. Since $g_{n}$ is an ideal in $a_{p}$, it follows from the equation (3.1.5) that $g$ is an ideal in 
$g^{B}$ and $g_{p}$ is an ideal in $g_{p}^{B}$ and that

$$
\alpha_{p}: g \rightarrow g_{p}
$$

Lemma 5. Let the notation be as above. Assume $M$ is simply connected. Then for any $p \in M$ the map $\alpha_{p}$ defines an isomorphism of $\mathrm{g}^{B}$ onto $\mathrm{g}_{p}^{B}$ and an isomorphism of $\mathrm{g}$ onto $\mathrm{g}_{p}$.

Proof. Given $b \in a_{p}, \quad v \in V_{p}$ it suffices to show that there exists $X \subseteq \mathrm{g}^{3}$ such that $\left(B_{X}\right)_{p}=b, X_{p}=v$. Let $o \in M$ and let $c$ be a piecewise differentiable curve joining $p$ and $o$. By solving the linear differential equations(3.1.3) and (3.1.5) along the curve $c$ with initial value $(b, v)$ we obtain at $o$ an element $\left(b^{\prime}, v^{\prime}\right) \in \mathfrak{g}_{o}$. The fact that $b_{o}$ in $\mathfrak{a}_{o}$ follows from (3.1.5) and the fact that $\xi_{q} \cong \mathfrak{a}_{q}$ for all points $q$. Assert that the element $\left(b^{\prime}, v^{\prime}\right)$ is independent of the curve $c$. To prove this it suffices to assume $c$ is a closed curve, that is $p=o$, and to show that $b=b^{\prime}$ and $v=v^{\prime}$. But now since $M$ is simply connected by a standard deformation argument it suffices to assume that $c$ lies in an arbitrarily small neighborhood $W$ of $p$ (recall that $p$ is an arbitrary point of $M$ and $(b, v)$ is an arbitrary element in $g_{p}$ ). But now, as noted above, $W$ can be chosen so that $\alpha_{p}\left(\mathrm{~g}_{w}^{k}\right)=\mathrm{g}_{p}^{\eta}$. It follows then that $b^{\prime}=b$ and $v^{\prime}=v$.

Now let $X \in \mathscr{T}^{1}(M)$ be the vector field defined by letting $X_{o}=v^{\prime}$. Now if $W^{\prime}$ is a neighborhood of $o$ such that $\alpha_{o}\left(\mathfrak{g}_{W^{\prime}}^{B}\right)=\mathfrak{g}_{o}^{B}$ then it is clear from the equations (3.1.3) and (3.1.5) that the restriction of $X$ to $W^{\prime}$ lies in $a_{W}^{R}$. This proves that $X$ is of class $C^{\infty},\left(B_{X}\right)_{o}=b^{\prime}$ and that $X \in \mathfrak{g}^{B}$. It also follows that $\alpha_{p}(X)$ $=(b, v)$.

3.4. An element $X \in \mathrm{g}^{B}$ will be called an infinitesimal transvection at $p$ if $\left(B_{X}\right)_{p}=0$. It is clear that if $X$ is an infinitesimal transvection at $p$ then $X \in g$. The following lemma is an immediate consequence of Lemma 5 .

Lemma 9. Assume $M$ is simply connected. Let $p \in M$. Let $m_{p} \subseteq g$ be the spare of all infinitesimal transvections at $p$ and let $\mathfrak{h}_{p} \subseteq \mathrm{g}$ be the set of all $X \in \mathrm{g}$ such that $X_{p}=0$. Then $\mathrm{m}_{p}=\alpha_{p}^{-1}\left(V_{p}\right)$ and $\mathfrak{h}_{p}=\alpha_{p}^{-1}\left(g_{p}\right)$ so that $\mathrm{g}=h_{p}+\mathrm{m}_{p}$ is a direct sum with $\mathfrak{h}_{p}$ a subalgebra of $\mathrm{g}$ and $\left[\mathfrak{h}_{p}, \mathrm{~m}_{p}\right] \subseteq \mathrm{m}_{p}$. Furthermore $\mathrm{m}_{p}$ generates 8. In fact $\mathrm{g}=\left[\mathrm{m}_{p}, \mathrm{~m}_{p}\right]+\mathrm{m}_{p}$ (non-direct in general).

The reason for restricting attention to $g$ instead of $g^{\prime \prime}$ from this point on is given in 
Lemma 7. Let the ideal $\mathrm{g}$ in the set of all infinitesimal B-affine transformations be defined by (3.3.1). Let $S$ be a tensor field on $M$. If $S$ is covariant constant with respect to $B$ then $S$ is invariant under $g$ (that is, $L_{X} S=0$ for all $X \in g)$.

Furthermore if $M$ is simply-connected then $S$ is covariant constant with respect to $B$ if and only if $S$ invariant under $g$.

Proof. Assume $\nabla_{Y}^{R} \mathrm{~S}=0$ for all $Y \in \mathscr{T}^{1}(M)$. But then for any $p \in M, S_{p}$ is invariant under the restricted holonomy group at $p$. Thus $b\left(S_{p}\right)=0$ for all $b \in B_{p}$. Now let $X \in g$. Then $\left(L_{X} S\right)_{p}=\left(\nabla_{Y}^{R} S\right)_{p}+\left(B_{X}\right)_{p} S_{p} . \quad$ But $\nabla_{X}^{R} S=0$ and since $\left(B_{X}\right)_{p} \in B_{p}$ it follows that $\left(B_{X}\right)_{p} S_{p}=0$. Hence $S$ is invariant under $g$. Now assume $M$ is simply connected and $S$ is invariant under $g$. Let $Y \in \mathscr{T}^{1}(M)$ and $p \in M$. Then by Lemma 5 there exists $X \in m_{p}$ such that $X_{p}=Y_{p}$. But $\left(S_{X}\right)_{p}=0$. Thus $\left(\nabla_{Y}^{R} S\right)_{p}=\left(\nabla_{X}^{B} S\right)_{p}=\left(L_{X} S\right)_{p}$. But $L_{X} S=0$. Thus $\left(\nabla_{Y}^{R} S\right)_{p}=0$ and hence $S$ is covariant constant.

Q.E.D.

Assuming only that $M$ is simply connected we now prove a part of Theorem 2 in the infinitesimal sense.

Lemma 8. Let $A$ be an affine connection on a manifold $M$. Assume that there exists an affine connection $B$ on $M$ such that (1) $A$ is rigid with respect to $B$ and (2) $B$ is invariant under parallelism. Let $g$ be the ideal in the Lie algebra of infinitesimal B-affine transformation defined by (3.3.1). Then $g$ is a Lie algebra of infinitesimal A-affine transformations.

Proof. Let $S=B-A$. By definition $S$ is covariant constant with respect to $B$. But then by Lemma $7 S$ is invariant under 9 . Writing $\nabla_{Y}^{A}=\nabla_{Y}^{R}-S_{Y}$ for any $Y \in \mathscr{T}^{1}(M)$ one immediately verifies $(3.1 .4)$ for any $X \in \mathfrak{g}$ when $A$ is substituted for $B$ in (3.1.4).

Q.E.D.

3.5. Now one knows that the group of all $B$-affine transformations of $M$ is a Lie group. See [2]. Let $G^{B}$ be the simply connected covering group of the identity component of this group. We may regard $G^{H}$ as operating on $M$ by passage to the group of $B$-affine transformations. It is clear then that we may identify the Lie algebra of $G^{B}$ with a subalgebra $g^{\prime}$ of $\mathrm{g}^{B}$. (See Note 1 in $\S 1$ ). Now $g \cap g^{\prime}$ is an ideal in $g^{\prime}$. Let $G$ be the subgroup of $G^{B}$ corresponding to $g \cap g^{\prime}$. Since $G^{b}$ is simply connected and $G$ is normal one knows that $G$ is also a simply connected closed subgroup of $G^{i 3}$ 
Now assume $M$ is simply connected. Then one knows that every element $X \in \mathrm{g}^{B}$ generates a one parameter subgroup of $B$-affine transformations, that is $\mathrm{g}^{\prime}=\mathrm{g}^{B}$, if and only if $M$ is complete with respect to $B$ and that in such a case $G^{B}$ is transitive on $M$. (See [4], p. 35 and [8], p. 77). We wish to observe now that in this statement $\mathrm{g}$ may be substituted for $\mathrm{g}^{B}$ and $G$ for $G^{B}$. Indeed if $M$ is complete then it is general theorem (see Prop. p. 67 in [8]) that every element in $g$ (and in fact in $g^{B}$ ) can be integrated. Conversely if $X \in \mathfrak{g}$ is an infinitesimal transvection at $p \in M$ and $X$ generates a one parameter group $g(t)$ then $g(t) \cdot p$ is the geodesic through $p$ with the tangent vector $X_{p}$ and it is defined for all values of the canonical parameter $t$. This is immediate since $\left(B_{X}\right)_{p}=0$ implies $\left(\nabla_{X} X\right)_{p}=\left(L_{X} X\right)_{p}=[X, X]_{p}=0$. Furthermore $L_{X}\left(B_{X}\right)=0$ so that $\left(B_{X}\right)_{q}=0$ for all $q=g(t) \cdot p$ with $t$ arbitrary. Finally to show that $G$ is transitive on $M$ it is enough to show that $G$ carries an arbitrary point into an open and closed set. This, however, is immediate under the condition of completeness since the group generated by an element $X \in \mathfrak{m}_{p}$ for (See Lemma 6) any $p \in M$ lies in $G$.

LEMMA 9. Let $B$ be an affine connection, which is invariant under parallelism, on a simply-connected manifold $M$. Let the group $G$ and the Lie algebra of vector fields $g$ be defined as above. Then $M$ is complete with respect to $B$ if and only if $\mathrm{g}$ is the Lie algebra of $G$.

Furthermore if $M$ is complete with respect to $B$ then $M$ is a reductive homogeneous space with respect to $G$. In fact if $p \in M$ and $H_{p} \subseteq G$ is the isotropy group at $p$ then $\mathrm{g}=\mathfrak{h}_{p}+\mathfrak{m}_{p}$ is direct sum where $\mathfrak{h}_{p}$ is the Lie algebra of $H_{p}$ and $\mathrm{m}_{p}$ is the space of infinitesimal transvections at $p$ and $\mathrm{m}_{p}$ is invariant under $A d H_{p} . \quad$ Moreover the linear group defined on the tangent space at $p$ by $H_{p}$ (this is equivalent to the action of $A d H_{p}$ on $\mathrm{m}_{p}$ ) is the homogeneous holonomy group at $p$.

One of the two statements of Theorem 2 is an immediate corollary of Lemmas 8 and 9 .

Theorem 4. Let $A$ be an affine connection on a simply connected manifold $M$. Assume that there exists a second affine connection $B$ on $M$ such that (1) $B$ is invariant under parallelism (2) $A$ is rigid with respect to $B$ and (3) $M$ is complete with respect to $B$. 
Let $\mathrm{g}$ be the Lie algebra of infinitesimal $B$-affine transformations $X$ on $M$ such that $\left(B_{X}\right)_{p} \in \exists_{p}$ for some (and hence every) point $p \in M$ where $s_{p}$ is the ( $B$ ) holonomy algebra at $p$. Then $M$ is a reductive homogeneous space with respect to a connected simply connected Lie group $G$ operating as A-affine (and also $B$-affine) transformations on $M$. Furthermore $G$ can be chosen so that $g$ is the Lie algebra of vector fields on $M$ defined by the action of $G$ (See Note 1 ).

4. The definition of $S$ on a reductive homogeneous space. 1. To prove the second statement of Theorem 2 we shall assume in this section that $M$ is a reductive homogeneous space with respect to a connected Lie group $G_{1}$. Let $g_{1}$ be the Lie algebra of $C_{1}$.

Now let $o \in M$ and let $H_{1} \subseteq G_{1}$ be the isotropy group at $o$ and let $h_{1}$ be the Lie algebra of $H_{1}$. By assumption there exists a subspace $\mathfrak{m}_{1} \subseteq g_{1}$ such that (a) $\mathfrak{g}_{1}=\mathfrak{h}_{1}+\mathfrak{m}_{1}$ is a direct sum and (b) $\mathfrak{m}_{1}$ is stable under $A d H_{1}$.

We will now show how the complement $\mathfrak{m}_{1}$ to $\mathfrak{h}_{1}$ defines a tensor field $S$ of type $\left(\begin{array}{c}i \\ j k\end{array}\right)$ on $M$ as soon as an affine connection is given on $M$.

Let $\gamma$ be defined as in Note $1, \S 1.2$. Let $g_{r}=\gamma\left(g_{1}\right)$. Since $r$ is a homomorphism it is clear that we may regard $A d G_{1}$ as operating on $g_{r}$. Now let $\mathfrak{h}=\gamma\left(\mathfrak{h}_{1}\right)$ and $m=\gamma\left(\mathfrak{m}_{1}\right)$. Since the kernel of $\gamma$ is contained in $\mathfrak{h}_{1}$ note that (1) $\gamma$ is faithful on $m_{1}$, (2) $g_{r}=\mathfrak{h}+m$ is a direct sum and (3) $m$ is stable under $A d H_{1}$.

For any point $p \in M$ let $V_{p}$ designate the tangent space at $p$ and let $\tau_{p}$ be the mapping

$$
\tau_{p}: \mathfrak{g}_{r} \rightarrow V_{p}
$$

defined by putting $\tau_{p} X=X_{p}$ where $X_{p}$ is the value of $X$ at $p$.

Now for each $p \in M$ define the subspace $m_{p} \subseteq \mathfrak{g}$ by the relation

$$
\mathrm{m}_{p}=\operatorname{Adg}(\mathrm{m})
$$

where $g \in G_{1}$ is such that $g \cdot o=p$. It is clear that $\mathfrak{m}_{p}$ depends only on the coset $g H_{1}$ and not solely on $g$. Now by (1) and (2) above it is clear that $\tau_{0}$ defines an isomorphism of $\mathrm{m}$ onto $V_{0}$. But since $A d g\left(H_{1}\right)$ is the isotropy group at $p$ where $g \cdot o=p$ it is clear, more generally, that the restriction of $\tau_{p}$ to $m_{p}$ is an isomorphism $m_{p}$ onto $V_{p}$. Let

$$
\varphi_{p}: V_{p} \rightarrow m_{p}
$$

be the map which is inverse to the restriction of $\tau_{p}$ to $m_{p}$. That is, $\varphi_{p}$ is such 
that $\tau_{p} \varphi_{p}$ is the identity on $V_{p}$.

Now assume that an affine connection $A$ is given on $M$ ( $A$ is not as yet assumed to be invariant under the action of $G)$. For any $X \in \mathscr{T}^{1}(M)$ let $A_{X}$ $\in \mathscr{L}(M)$ be defined by

$$
A_{X}=L_{X}-\nabla_{X}^{A} .
$$

Now if $Y \in \mathscr{T}^{1}(M)$ let $S_{Y} \in \mathscr{L}(M)$ be defined by

$$
\left(S_{Y}\right)_{p}=\left(A_{\varphi p\left(Y_{p}\right)}\right)_{p}
$$

where $Y_{p} \in V_{p}$ is the value of $Y$ at $p$. It is obvious that the mapping $Y \rightarrow S_{Y}$ (unlike $X \rightarrow A_{X}$ ) is a $\mathscr{T}^{0}(M)$-linear from $\mathscr{T}^{1}(M)$ into $\mathscr{L}(M)$ and hence defines a tensor $S$ of type $\left(\begin{array}{c}i \\ j k\end{array}\right)$.

We now define a new affine connection $B$ on $\mathrm{M}$. The connection $B$ is such that $B-A=S$. That is $B$ is given by the relation

$$
\nabla_{Y}^{B}=\nabla_{Y}^{A}+S_{Y}
$$

for all $Y \in \mathscr{T}^{1}(M)$. The principal relation between the affine connection $B$ and the action of $G_{1}$ on $M$ which concerns us now is given in

Lemma 10. Let $M, G_{1}$ and $B$ be as above. Then any tensor field on $M$ which is invariant under $G_{1}$ is covariant constant with respect to $B$.

Proof. This is practically immediate from the definition of $S$. Assume $U$ is a tensor field on $M$ which is invariant under $G_{1}$. That is $L_{X} U=0$ for all $X \in g_{\text {r. Now let }} Y \in \mathscr{T}^{1}(M)$ and $p \in M$. Let $X=\varphi_{p} Y_{p}$. Then

$$
\begin{aligned}
\left(\nabla_{Y}^{B} U\right)_{p} & =\left(\nabla_{Y}^{A} U\right)_{p}+\left(S_{X} U\right)_{p} \\
& =\left(\nabla_{X}^{A} U\right)_{p}+\left(A_{X} U\right)_{p} \\
& =\left(L_{X} U\right)_{p} \\
& =0
\end{aligned}
$$

and hence $U$ is $B$-covariant constant.

Q.E.D.

Now assume that the elements of $G_{1}$ act as $A$-affine transformations of $M$. One knows that this is the case if and only if

$$
\left[L_{X}, \nabla_{Y}^{A}\right]=\nabla_{[X, Y]}^{A}
$$

for all $X \in \mathfrak{g}_{r}, Y \in \mathscr{T}^{1}(M)$.

Lemma 11. Let $M, G_{1}$ and $A$ be defined as above. Let the tensor field $\cdot S$ 
on $M$ be defined by (4.1.2). Then if $G_{1}$ acts as a group of A-affine transformation $S$ is invariant under the action of $G_{1}$.

Proof. Let $g \in G_{1}$. If $U \in \mathscr{T}(M)$ or $\mathscr{D}(M)$ we let $g \cdot U$ be the tensor field or derivation into which $U$ is carried under the diffeomorphism of $M$ defined by $g$. To prove the lemma it suffices to show that $g \cdot S_{Y}=S_{g \cdot Y}$ for all $Y \in \mathscr{T}^{1}(M)$ and $g \in G_{1}$. In fact it suffices to show that if $g \cdot o=p$

$$
\left(g \cdot S_{X}\right)_{p}=\left(S_{g} \cdot X\right)_{p}
$$

for all $g \in G, X \in \mathfrak{m} \subseteq \mathfrak{g}_{\mathrm{r}}$. But if $X \in \mathfrak{m},\left(S_{X}\right)_{0}=\left(A_{X}\right)_{0}$ where $A_{X}$ is defined by (4.1.1). Hence $\left(g \cdot S_{X}\right)_{p}=\left(g \cdot A_{X}\right)_{p}$. But since $g$ defines an $A$-affine transformation $\nabla_{g \cdot X}^{A}=g \cdot V_{X}^{A}$ and in any case $L_{g \cdot X}=g \cdot L_{X}$. Thus $g \cdot A_{X}=A_{g \cdot x}$. Consequently to prove the lemma it suffices only to show that $\left(S_{g \cdot X}\right)_{p}=\left(A_{g} \cdot x\right)_{p}$ for any $X \in m$. But to show this it suffices to show that $g \cdot X \in \mathfrak{m}_{p}$. But this is immediate since $g \cdot X=A d g X$.

Q.E.D.

TheOREM 5. Let $M$ be a manifold provided with an affine connection $A$. Assume that $M$ is a reductive homogeneous space with respect to a connected Lie group $G$ acting as A-affine transformations on $M$. Let $B$ be the affine connection on $M$ defined by (4.1.3). Then (1) $B$ is invariant under parallelism (2) $A$ is rigid with respect to $B$ and (3) $M$ is complete with respect to $B$.

Furthermore if $g$ is defined by (3.3.1), with respect to $B$ then for any point $p$ the map (3.3.2) is an isomorphism onto. Moreover $\mathfrak{g} \subseteq g_{\mathrm{r}}$ where $g_{r}$ is defined as in $\S 4.1$. In fact if $p$ is any point of $M$ then $m_{p}$ defined by (4.1.0) is the set of all infinitesimal $(B)$ transvections at $p$ (in other wora's the definition of $m_{p}$ in $\S 4.1$ is consistent with the definition of $m_{p}$ in $\S 3.4$ ) and $g=\left[m_{p}, m_{p}\right]+m_{p}$.

Proof. By Lemmas 10 and $11 S$ is covariant constant with respect to $B$. That is, $B$ is rigid with respect to $A$. On the other hand since $S$ is invariant with respect to $A$. On the other hand since $S$ is invariant with respect to $G_{1}$ it is obvious that $\left[L_{X}, \nabla_{Y}^{R}\right]=\nabla_{[X, Y]}^{R}$ for all $X \in \mathrm{g}_{\Upsilon}$ and $Y \in \mathscr{T}^{1}(M)$. Thus $B$ is invariant under the action of $G_{1}$. But this implies $R^{B}$ and $T^{B}$ are also invariant under $G_{1}$. But then by Lemma $10 R^{B}$ and $T^{B}$ are covariant constant with respect to $B$. That is $B$ is invariant under parallelism. Now if $m_{p}$ is defined by (4.1.0) and $X \in m_{p}$ is clear that $B_{X}=L_{X}-\nabla_{X}^{R}$ vanishes at $p$. That is $X$ is a $B$ infinitesimal transvection at $p$. Thus $m_{p} \subseteq \Omega$ and by Lemma $6 \alpha_{p}$ maps $\Omega$ onto 
$g_{p}$ and $g=\left[m_{p}, m_{p}\right]+m_{p}$. But now as in $\S 3.5$ to prove $M$ is complete with respect to $B$ it suffices to show that every element $X \in g$ generates a oneparameter group of diffeomorphisms of $M$. But $X_{1} \in g_{1}$ exists such that $\gamma\left(X_{1}\right)$ $=X$. It is clear then that the action of $\exp t X_{1}$ on $M$ is such a one parameter group.

Q.E.D.

\section{REFERENCES}

[1] W. Ambrose and I. M. Singer, On homogeneous Riemannian manifolds, Duke Mathematical Journal, 25 (1950), pp. 647-669.

[2] J. Hano and A. Morimoto, Note on the group of affine transformations of an affinely connected manif sd, Nagoya Mathematical Journal, 8 (1955), pp. 71-81.

[3] J. Hano, On affine transformations of a Riemannian manifold, Nagoya Mathematical Journal, 9 (1955), pp. 99-109.

[4] S. Kobayashi, Espaces à connexions affine et Riemanniennes symétriques, Nagoya Mathematical Journal, 9 (1955), pp. 25-37.

[5] S. Kobayashi, A theorem on the affine transformation group of a Riemannian manifold, Nagoya Mathematical Journal, 9 (1955), pp. 39-41.

[6] B. Kostant, Holonomy and the Lie algebra of infinitesimal motions of a Riemannian manifold, Transactions of the American mathematical Society, 80 (1955), pp. 528-542.

[7] K. Nomizu, Invariant affine connections on homogeneous spaces, American Journal of Mathematics, 76 (1954), pp. 33-65.

[8] K. Nomizu, Lie groups and differential geometry. Publications of the Mathematical Society of Japan, Tokyo, 1956.

University of California 